\title{
Patient Experiences of Postinfectious Olfactory Dysfunction
}

\author{
Carl M Philpott ${ }^{a, b, c}$ James Boardman ${ }^{c}$ Duncan Boak ${ }^{c}$ \\ aThe Norfolk Smell \& Taste Clinic, The Norfolk \& Waveney ENT Service, Norwich, UK; borwich Medical School, \\ University of East Anglia, Norwich, UK; 'Fifth Sense, Phoenix Business Centre, Unit 8, Barrow-in-Furness, UK
}

\section{Keywords}

Olfactory dysfunction - Postinfectious olfactory

dysfunction - Post-viral olfactory loss · Anosmia · Parosmia .

Therapeutics

\section{Abstract}

Introduction: To highlight the importance of the need for new treatment modalities, this study aimed to characterise the experience of patients with postinfectious olfactory dysfunction (PIOD) in terms of the treatment they received. Methods: An online survey was hosted by the Norwich Clinical Trials Unit on the secure REDCap server. Members of the charity Fifth Sense (the UK charity that represents and supports people affected by smell and taste disorders) were invited to participate. Results: There were 149 respondents, of whom 127 had identified themselves as having (or had) PIOD. The age range of respondents to the survey was $28-85$ years, with a mean of $58 \pm 12$ years, with the duration of their disorder $<5$ years in $63 \%$ of cases. Respondents reported experiencing variable treatment with oral and/or intranasal steroids given typically (28\%), often with no benefit, but with $50 \%$ receiving no treatment whatsoever; only $3 \%$ reported undertaking olfactory training. Over two-thirds of patients experience parosmia and, up to 5 years from the onset of the problem, were still actively seeking a solution. Conclusion: There appears to be a need to encourage greater use of guidelines for olfactory disorders amongst medical practitioners and also to develop more effective treatments for patients with PIOD, where there is clearly an unmet need.

(c) 2021 S. Karger AG, Basel

\section{Introduction}

Loss of smell is a common complaint in adults and yet has been underestimated. Anosmia, complete loss of smell, is thought to affect at least $1-5 \%$ of the population [1]. Based on European estimates [2], anosmia is more prevalent than profound hearing loss or blindness in the UK as data from the Royal National Institutes for the Blind and the Deaf show that 2 million (3\%) are affected by sight loss [3] and 900,000 (1.3\%) are affected by profound hearing loss [4]. Causes for olfactory loss vary, but the main diagnostic groups include sinonasal disease (62\%) and postinfectious olfactory dysfunction (PIOD) (11\%) [5]. Olfactory dysfunction has now emerged as a key symptom in the coronavirus pandemic, and it is pos-
Correspondence to:

Carl M. Philpott, c.philpott@uea.ac.uk 
sible we may see a cohort of patients with lasting problems following the peak of the COVID-19 impact [6-8]. Historically, there is wide variation in clinical practice reported by clinicians to patients on prognosis or treatment [9]. Guidelines for the overall management of olfactory disorders now exist in the Position Paper on Olfactory Dysfunction [1], and recently, the Clinical Olfactory Working Group has produced a consensus statement on managing PIOD [10], but the impact of those guidelines has yet to penetrate routine clinical practice.

Previously published data have shown the high impact on the quality of life experienced by olfactory disorder patients, including depression, anxiety, impairment of eating experience, feelings of isolation, and relationship difficulties [11, 12]. Most patients suffer a loss of flavour perception, which can adversely affect their appetite [13], but this can be made even worse when parosmia is also present. Given the number of potential treatment options for PIOD that has been proposed but yet to undergo formal evaluation through a randomised clinical trial [14], there is a pressing need to deliver more RCTs in this area. A recent German study has highlighted topical vitamin A as a potential therapeutic agent for further evaluation [15]. In anticipation of setting up a clinical trial to evaluate the role of vitamin A intranasally for PIOD, we sought to generate evidence of the need for funders through public and patient involvement in partnership with the charity Fifth Sense that represents patients affected by smell and taste disorders in the UK (www.fifthsense.org.uk). The aim of this study was to characterise the experience of patients with PIOD in terms of the treatment they received in order to demonstrate any unmet need.

\section{Materials and Methods}

A survey was set up by the Norwich Clinical Trials Unit in conjunction with Fifth Sense and was designed by the authors (a clinician and 2 patients with olfactory dysfunction). This was uploaded on the REDCap server at the Norwich Clinical Trials Unit; REDCap is a secure Web application for building and managing online surveys and databases. A secure link was then circulated to members of Fifth Sense via e-mail as part of the public and patient involvement in the proposed trial application. The questions were designed to be applicable to PIOD sufferers in the UK and beyond. The survey was live for 2 months at the beginning of 2019. As this study was considered service evaluation, no ethical approval was needed according to the UK Health Research Authority guidelines [16].

\section{Eligibility Criteria}

The survey was sent to members of Fifth Sense who had identified themselves as joining the charity for having suffered olfactory dysfunction following an upper respiratory tract infection; participants had to recall symptoms of olfactory dysfunction following directly by an upper respiratory tract infection. Responses were screened to remove anyone not identifying themselves with PIOD and also to document coexisting sinonasal disorders including chronic rhinosinusitis (CRS), allergic rhinitis, and nasal septal deviation which would be considered exclusion criteria in any subsequent trial.

\section{Survey Questions}

Only the questions that did not refer specifically to involvement in a clinical trial are listed here.

1. Do you have a reduced sense of smell because of a bad cold/ infection you suffered? If no, you can stop the survey here.

2. Did you suffer the cold/infection $<5$ years ago? $>5$ years ago?

3. Have you suffered from smell distortions (parosmia) or smell hallucinations (phantosmia)?

4. Have you received any treatment to date?

5. What treatment did you receive? (free text)

6. - CRS with or without nasal polyps

- Allergic rhinitis (hay fever)

- Severe deviation of the nasal septum

- I am using nasal medications at the moment

7. Do you have any comments about smell loss due to a bad cold?

8. If you are willing, please tell us your age.

\section{Analysis}

Given the nature of the study, no formal statistical analysis was undertaken; rather a descriptive narrative of the survey findings is presented including representative comments left by some patients.

\section{Results}

There were 149 Fifth Sense members who responded to the survey, of whom 127 declared they had experienced PIOD. In the 127 PIOD respondents, 13 reported both CRS and AR, 18 AR, and 13 CRS. Seven reported a severe septal deviation. The age range was $28-85$ years, with a mean of $57 \pm 11$ years. The duration of their disorder was $<5$ years in $63 \%$ of cases, with $64 \%$ reporting the presence of parosmia. In terms of treatment received, $50 \%$ had not received any treatment at all from their GP or ENT specialists; 24 members reported they were currently using nasal medications. Prior medication use included oral and intra-nasal corticosteroids, most commonly given in $28 \%(n=36)$. Additionally, antibiotics were received by $5 \%$ of patients, despite a lack of evidence to support this, and only $3 \%$ of participants reported olfactory training as a treatment they had been advised to perform. Figure 1 shows the treatments reported by respondents (some respondents reported $>1$ treatment). Twenty-four patients (without other reported sinonasal disorders) (19\%) also reported having undergone MRI and/or CT scans. 
Fig. 1. Prior medication use by respondents (number of respondents for each medication) - some respondents reported in $>1$ category. OCS, oral corticosteroids; INCS, intranasal corticosteroids; ND, nasal douching; Abx, antibiotics.

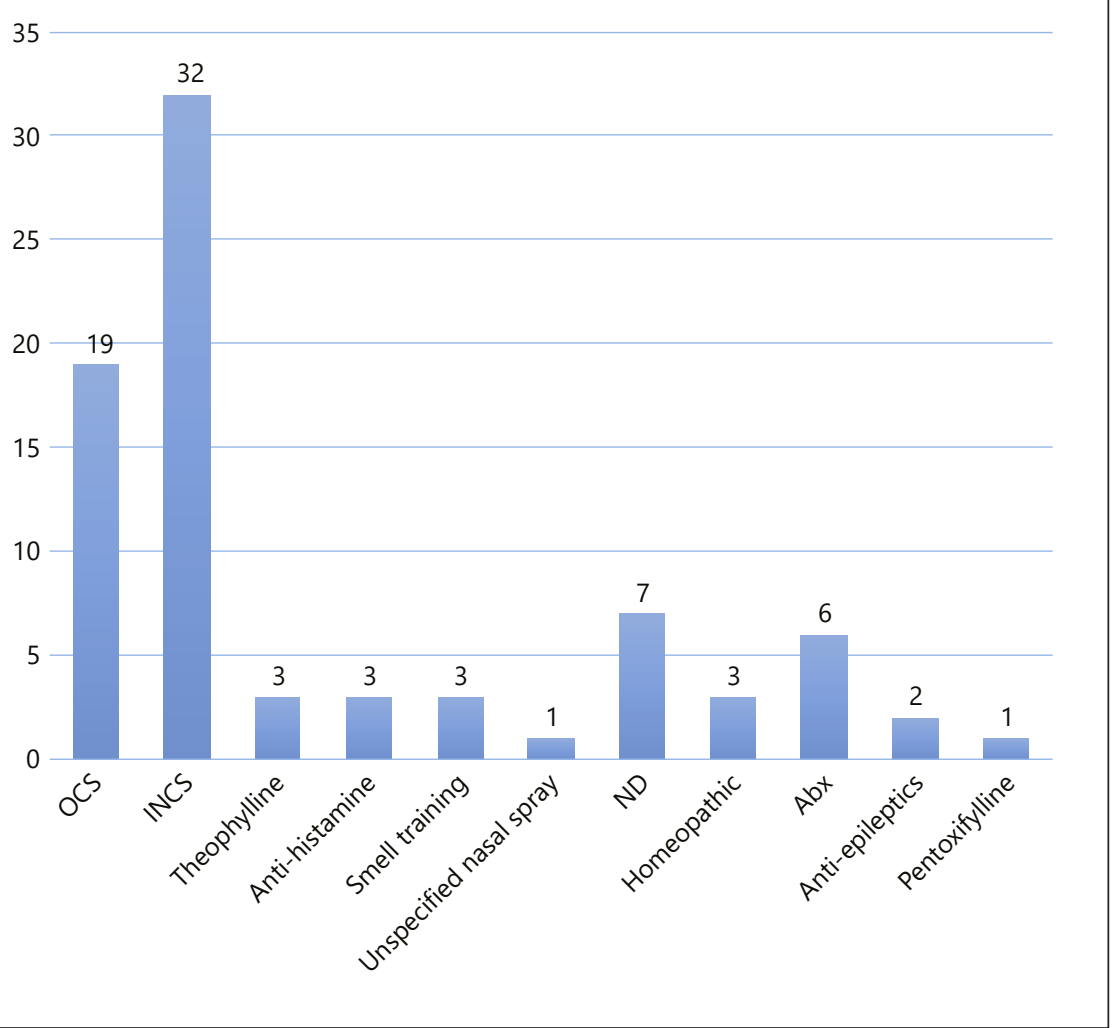

\section{Quotations from Survey Participants}

Participants were asked to leave comments, and many did. Here are a few examples: "I am very low with the effects of PIOD has had on my life; it affects my partner too: "I lost my very sensitive sense of smell suddenly after a really bad infection. I went to my GP a few months later, but he said bad luck. He did not refer me to an ENT specialist, so I do not know if I have any of the medical conditions mentioned. The only thing I can smell consistently is orange peel. Very occasionally, I get a brief 'glimpse' of another smell, for example, fish or carnations."

"It was a severe cold when I lost my voice completely twice. I was shocked that my smell went and keep hoping it will come back. Even though I saw an ENT consultant and tried steroids no change."

"It was a prolonged dose of bad cold then flu in the run up to Christmas 2017 that finally wiped mine out. I could taste something when I started eating my Christmas dinner but by the end of the meal I couldn't taste or smell anything. Since then, its improved a little but only a little and something has really left my life. It's taken the colour from day-to-day life."

Patient Experiences of Postinfectious Olfactory Dysfunction
"I have only ever had the flu twice in my life, with the last bout in January 2018 resulting in the complete loss of my sense of taste and smell. I still remember the point at which the loss occurred. It was a serious flu, around the time the Ozzy Flu epidemic was rife in the area I live. The virus left me bed ridden for a few days and it was around the $4 / 5$ day where the virus was at its worst that I remember a complete blockage occurring where I struggled for breath, and after this point I have been unable to taste or smell anything."

"Lost smell and taste 7 months ago following flu, have had a very negative response from the medical profession including 1 doctor who was extremely rude to me."

\section{Discussion}

The survey helps delineate the paucity of effective treatment provision for this particular group of patients with olfactory dysfunction. It is particularly notable that only $3 \%$ of survey participants reported undertaking olfactory training as a treatment option which, given the increasing volume of evidence to support this option [17], 
is surprising. This may well be a product the current knowledge of their physicians who treated them Furthermore, it is interesting that some patients underwent imaging which would not ordinarily indicate if the history is clear, and endoscopy shows no evidence of intranasal pathology [1]; this suggests that work is needed to disseminate the guidelines from the 2017 Position Paper on Olfactory Dysfunction. Although PIOD represents a smaller percentage of the causes of olfactory disorders overall than CRS [5], the latter condition benefits from greater attention, and PIOD patients typically make up a higher percentage of attendees in a specialist clinic (typically about 25\%) [18]. Managing smell loss has previously been identified as a source of frustration for clinicians in otorhinolaryngology, in part due to the lack of therapeutic options to deploy [9] but can often lead to dismissal of patients $[12,19]$. A number of medical and complementary medicine options have been studied in case series, but only few previous have been subjected to the scientific rigour of a randomised controlled trial [14]. A recent position article on olfactory dysfunction has outlined the utility of smell training [1], but despite the increasing uptake of this option through information provided on the Fifth Sense Website, significant numbers of patients remain markedly affected. It is perhaps the apparent dearth of therapeutic agents that are deemed effective by clinicians that leads to the picture we see here of half the patients reporting a lack of treatment; certainly $93 \%$ of participants expressed a strong willingness to participate in any future trials. Other studies have charted the experience of those with olfactory disorders [20-22], but guidelines have been only more recently developed that help guide clinicians on generic management and specific treatment recommendations $[1,10,23]$; therefore, medical practitioners are perhaps likely to remain unfamiliar with the best approach to management.

This survey has its limitations. Firstly, it may be biased in its respondents being those who have a persistent olfactory disturbance; however, these are the patients who present to doctors seeking further help and for whom any firstline treatment has failed. Secondly, the participants have self-reported their PIOD and any sinonasal disorders; however, our previous work has shown that self-reporting from Fifth Sense members tends to reflect the proportions of doctor-diagnosed cases seen in a specialist smell and taste clinic [24], so we do believe the respondents here are likely to reasonably accurately reflect a genuine group of PIOD patients. We did not however capture any information on who their prior medical contact was with such as GP, otorhinolaryngologist, or specialist olfactologist.
Post-viral olfactory loss typically occurs following viral injury to the olfactory epithelium which leaves the olfactory sensory neurones lacking in cilia and effectively nonfunctional [25], although the current COVID-19 may wreak its havoc on the olfactory system by different mechanisms [8]. Treatment of this condition does require some insights into the pathophysiology in order to target potential pathways where therapeutic agents can act. It may be possible that stem cell therapy yields some hope in this area in the future. Presently, topical treatments including theophylline [26], vitamin A [15], and sodium citrate [27-29] have shown some promise and should be considered amongst potential candidates for more rigorous scientific evaluation.

\section{Conclusion}

We hope that this survey's findings will provide a platform for more research, including clinical trials, to be conducted in the area of PIOD by demonstrating evidence of a need in patients persistently affected. We also hope that it serves as a reminder to clinicians encountering these patients to consider updating their knowledge of managing olfactory disorders, both in PIOD and other causes of olfactory dysfunction, especially in light of the current guidelines such as those produced by the Clinical Olfactory Working Group.

\section{Acknowledgements}

The authors thank Antony Colles for setting up the survey on the REDCap server at Norwich CTU.

\section{Statement of Ethics}

The data on which the study is based were gathered by Fifth Sense via an anonymous survey circulated amongst its beneficiaries and via social media. As a small charity, Fifth Sense does not have a research ethics committee nor is it required by the regulatory body (Charities Commission). However, the board of trustees had full oversight of the survey and gave full approval for the views of their beneficiaries to be captured in this way. The survey was also considered to be a service evaluation under HRA guidance [16].

\section{Conflict of Interest Statement}

There is no declared conflict of interest. 


\section{Funding Sources}

There is no funding for this study.

\section{Author Contributions}

Duncan Boak made substantial contributions to the conception or design of the work, the acquisition, analysis, and interpretation of data for the work and revised the work critically for important intellectual content and approved the final version to be published. Jim Boardman made substantial contributions to the conception or design of the work and approved the final version to be published. Carl Philpott made substantial contributions to the conception or design of the work, the acquisition, analysis, and interpretation of data for the work and revised the work critically for important intellectual content and approved the final version to be published. He is also in agreement to be accountable for all aspects of the work in ensuring that questions related to the accuracy or integrity of any part of the work are appropriately investigated and resolved.

\section{References}

1 Hummel T, Whitcroft KL, Andrews P, Altundag A, Cinghi C, Costanzo RM, et al. Position paper on olfactory dysfunction. Rhinology. 2017;54:1-30.

2 Landis BN, Konnerth CG, Hummel T. A study on the frequency of olfactory dysfunction. Laryngoscope. 2004 Oct;114(10):1764-

3 Prevalence of sight loss. Royal National Institute for the Blind; 2021.

4 Prevalence of profound hearing loss. Royal National Institute for the Deaf; 2021.

5 Damm M, Temmel A, Welge-Lüssen A, Eckel HE, Kreft MP, Klussmann JP, et al. [Olfactory dysfunctions. Epidemiology and therapy in Germany, Austria and Switzerland]. HNO. 2004 Feb;52(2):112-20.

6 Haehner A, Draf J, Dräger S, de With K, Hummel T. Predictive value of sudden olfactory loss in the diagnosis of COVID-19. ORL J Otorhinolaryngol Relat Spec. 2020;82(4): 175-80.

7 Parma V, Ohla K, Veldhuizen MG, Niv MY, Kelly CE, Bakke AJ, et al. More than smellCOVID-19 is associated with severe impairment of smell, taste, and chemesthesis. Chem Senses. 2020 Oct; 45(7):609-22.

8 Philpott C. Coronavirus: loss of smell and taste reported as early symptoms of COVID-19. The Conversation. 2020.

9 McNeill E, Ramakrishnan Y, Carrie S. Diagnosis and management of olfactory disorders: survey of UK-based consultants and literature review. I Laryngol Otol. 2007 Aug;121(8): 713-20.

10 Addison AB, Wong B, Ahmed T, Macchi A, Konstantinidis I, Huart C, et al. Clinical olfactory working group consensus statement on the treatment of post infectious olfactory dysfunction. J Allergy Clin Immunol. 2021 Jan.
11 Philpott CM, Boak D. The impact of olfactory disorders in the United kingdom. Chem Senses. 2014 Oct;39(8):711-8.

12 Erskine SE, Philpott CM. An unmet need: patients with smell and taste disorders. Clin Otolaryngol. 2020 Mar;45(2):197-203.

13 DeVere R. Disorders of Taste and Smell. Continuum. 2017 Apr;23(2):421-46.

14 Addison A, Philpott CM. A systematic review of therapeutic options for non-conductive olfactory dysfunction. The Otorhinolarnyngologist. 2018;11(2):61-71.

15 Hummel T, Whitcroft KL, Rueter G, Haehner A. Intranasal vitamin A is beneficial in postinfectious olfactory loss. Eur Arch Otorhinolaryngol. 2017 Jul;274(7):2819-25

16 HRA Decision Tool. Health Research Authority.

17 Kattar N, Do TM, Unis GD, Migneron MR, Thomas AJ, McCoul ED. Olfactory training for postviral olfactory dysfunction: systematic review and meta-analysis. Otolaryngol Head Neck Surg. 2021 02;164(2):244-54.

18 Philpott C. Smell and taste disorders in the UK: first experiences with a specialised smell and taste outpatient clinic. Bulletin. 2014, 96(5):156-9.

19 Ball S, Boak D, Dixon J, Carrie S, Philpott C. Barriers to accessing healthcare in patients with olfactory and gustatory disorders. Authorea. 2020.

20 Brämerson A, Nordin S, Bende M. Clinical experience with patients with olfactory complaints, and their quality of life. Acta Otolaryngol. $2007 \mathrm{Feb}$;127(2):167-74.

21 Nordin S, Brämerson A. Complaints of olfactory disorders: epidemiology, assessment and clinical implications. Curr Opin Allergy Clin Immunol. $2008 \mathrm{Feb} ; 8(1): 10-5$
22 Chen G, Wei Y, Miao X, Li K, Ren Y, Liu J. Clinical features of olfactory disorders in patients seeking medical consultation. Med Sci Monit. 2013 Jun;19:444-50.

23 Hopkins C, Alanin M, Philpott C, Harries P, Whitcroft K, Qureishi A, et al. Management of new onset loss of sense of smell during the COVID-19 pandemic - BRS consensus guidelines. Clin Otolaryngol. 2021 Jan;46(1): 16-22.

24 Langstaff L, Pradhan N, Clark A, Boak D, Salam M, Hummel T, et al. Validation of the olfactory disorders questionnaire for Englishspeaking patients with olfactory disorders. Clin Otolaryngol. 2019 Sep;44(5):715-28.

25 Moran DT, Jafek BW, Eller PM, Rowley JC. Ultrastructural histopathology of human olfactory dysfunction. Microsc Res Tech. 1992 Oct;23(2):103-10.

26 Henkin RI, Schultz M, Minnick-Poppe L. Intranasal theophylline treatment of hyposmia and hypogeusia: a pilot study. Arch Otolaryngol Head Neck Surg. 2012 Nov;138(11):106470.

27 Whitcroft KL, Merkonidis C, Cuevas M, Haehner A, Philpott C, Hummel T. Intranasal sodium citrate solution improves olfaction in post-viral hyposmia. Rhinology. 2016;54(4): 368-74.

28 Philpott CM, Erskine SE, Clark A, Leeper A, Salam M, Sharma R, et al. A randomised controlled trial of sodium citrate spray for nonconductive olfactory disorders. Clin Otolaryngol. 2017 Dec;42(6):1295-302.

29 Whitcroft KL, Ezzat M, Cuevas M, Andrews $\mathrm{P}$, Hummel T. The effect of intranasal sodium citrate on olfaction in post-infectious loss: results from a prospective, placebo-controlled trial in 49 patients. Clin Otolaryngol. 2017 Jun;42(3):557-63. 